\title{
BMJ Open Prognostic significance of tissue factor in patients with pancreatic cancer: a systematic review protocol
}

\section{Haiyuan Li, ${ }^{1,2}$ Yang Yu (D) , ${ }^{1,2}$ Qianling Shi, ${ }^{3}$ Xueping Chen, ${ }^{4}$ Peng Zheng, ${ }^{1,2}$ Dengfeng Wang, ${ }^{1,2}$ Pengxian Tao, ${ }^{1,2}$ Baohong Gu, ${ }^{1,2}$ Xuemei Li, ${ }^{1,2}$ Tao Zhang, ${ }^{1,2}$ Lin Xiang, ${ }^{1,2}$ Dayong Xi, ${ }^{1,2}$ Lei Gao, ${ }^{1,2}$ Paul Maswikiti Ewetse, ${ }^{1,2}$ Hao Chen (D) ${ }^{1}$}

To cite: Li H, Yu Y, Shi Q, et al. Prognostic significance of tissue factor in patients with pancreatic cancer: a systematic review protocol. BMJ Open 2020;10:e037431. doi:10.1136/ bmjopen-2020-037431

- Prepublication history for this paper is available online. To view these files, please visit the journal online (http://dx.doi org/10.1136/bmjopen-2020037431).

HL and YY contributed equally.

HL and YY are joint first authors.

Received 03 February 2020

Revised 11 May 2020

Accepted 28 July 2020

Check for updates

(C) Author(s) (or their employer(s)) 2020. Re-use permitted under CC BY-NC. No commercial re-use. See rights and permissions. Published by BMJ.

${ }^{1}$ Department of Tumor Surgery, Lanzhou University Second Hospital, Lanzhou, China

${ }^{2}$ The Second Clinical Medical College, Lanzhou University, Lanzhou, China

${ }^{3}$ The First Clinical Medical College, Lanzhou University, Lanzhou, China

${ }^{4}$ Sleep Medicine Center, Gansu Provincial Hospital, Lanzhou, China

Correspondence to

Professor Hao Chen;

ery_chenh@|zu.edu.cn

\section{ABSTRACT}

Introduction Pancreatic cancer is a highly aggressive digestive system tumour with poor prognosis. Venous thromboembolism (VTE) is a well-known complication of pancreatic cancer, and tissue factor (TF) contributes to the generation of a hypercoagulable state and thrombotic disease in pancreatic cancer. Several studies showed that an elevated TF level was related to the development of VTE and influenced the survival of patients with pancreatic cancer. Thus, we wish to conduct a systematic review of literature to clarify the prognostic significance of TF in pancreatic cancer.

Methods and analysis Studies comparing the circulating microparticle-associated TF (MP TF) level between patients who had pancreatic cancer with and without VTE will be included to evaluate the roles of TF in VTE development. Studies comparing the survival data between patients with high TF expression and low TF expression will also be included to explore the association of TF expression with patient survival. The outcomes are plasma MP TF level and survival endpoints (overall and progressionfree survival), respectively. Primary studies of any type published in English will be included. Two reviewers will search Medline, EMBASE and Cochrane databases from inception to June 2020, retrieve relevant studies, and independently select the literatures and extract data from the included studies. The quality of each included study will be assessed by the Newcastle-Ottawa Scale score. The HR and $95 \% \mathrm{Cl}$ of each study will be pooled for survival outcome, and the standardised mean difference (SMD) with $95 \%$ Cls will be used for continuous outcomes. If meta-analysis is inappropriate, the result will only be reported qualitatively. Subgroup and sensitivity analyses will be considered to identify sources of heterogeneity. The Grades of Recommendation, Assessment, Development and Evaluation method will be applied to assess the level of evidence of this systematic review.

Ethics and dissemination There are no concerning ethical issues. The results will be published. PROSPERO registration number CRD42019133665.

\section{INTRODUCTION}

Pancreatic cancer is a type of highly aggressive digestive system tumour with poor prognosis. ${ }^{1}$ It is estimated that pancreatic cancer will be the second largest cancer-related
Strengths and limitations of this study

- A detailed and completed search strategy will be used to collect potentially eligible studies.

- Sources of heterogeneity will be carefully considered, and multiple solutions will be considered.

- The included literature will be limited to studies published in English.

- Heterogeneity among the tissue factor measuring methods may exist.

- The findings are potentially subject to publication bias.

cause of death in the USA by $2030 .^{2}$ Pancreatic cancer has insidious onset, rapid progression, low rate of early diagnosis and radical resection. ${ }^{3}$ Most of the patients already have advanced or distant metastasis at the first diagnosis. Despite recent advances in surgical techniques as well as chemotherapy and radiation therapy, patients with pancreatic cancer still has a dismal 5-year survival rate. ${ }^{3}$

Venous thromboembolism (VTE), associated with the generation of an intrinsic hypercoagulable state, is a well-known complication of malignant tumour. Sproul first discovered that patients with pancreatic cancer are prone to VTE in $1938 .{ }^{4}$ Subsequently, the reported incidence of VTE in patients with pancreatic cancer ranges from $17 \%$ to $57 \% .^{5}$ Several factors and pathological mechanisms contribute to the generation of a hypercoagulable state and VTE in pancreatic cancer. ${ }^{6}$ Of prime importance is tissue factor (TF), a single chain transmembrane-receptor glycoprotein with a molecular weight of approximately $47 \mathrm{kDa}$, which initiates the extrinsic pathway of coagulation. ${ }^{7}$ In recent years, it was reported that TF is expressed in many malignant tumour tissues, including pancreatic cancer, leading to increased blood coagulability and a high risk of VTE. ${ }^{89}$ In addition, high level of the plasma TF was detected in 
patients with pancreatic cancer. ${ }^{10}$ Pancreatic cancer cells express TF and release microparticle-associated TF (MP TF) into blood. ${ }^{11} 12$

Several studies have found that thrombosis and diffuse intravascular coagulation are associated with poor prognosis in patients with pancreatic cancer. ${ }^{13-17}$ Meanwhile, substantial evidence indicated that $\mathrm{TF}$ is of predictive value in the biological features of tumours and the survival of patients. It was found that TF plays an important role in promoting angiogenesis, ${ }^{18}$ inflammatory reaction, ${ }^{19}$ tumour invasion ${ }^{20}$ and metastasis. ${ }^{21}$ Compared with tumour cells with a higher degree of differentiation in pancreatic cancer, tumour cells with lower degree of differentiation seem to have higher TF expression and associated with decreased overall survival (OS). ${ }^{9}$ 22-24 Therefore, we hypothesise that the elevated circulating TF level may contribute to the development of VTE, and TF expression in tumour tissue may be associated with poor prognosis in patients with pancreatic cancer. To test this hypothesis, we plan to undertake a systematic review of previous studies assessing the association between TF and pancreatic cancer.

\section{METHODS AND ANALYSIS \\ Objective of the review}

This review aims to clarify the prognostic significance of TF in pancreatic cancer. This review contains two sections. First, we will evaluate the association between circulating MP TF level and VTE. Second, we will explore the impact of TF expression on patients' survival.

\section{Eligibility criteria}

Participants

Patients with pancreatic cancer are eligible for the review. The diagnosis will be pathologically confirmed. There are no limitations on the stages, grades and types of pancreatic cancer. Patients will be excluded if they have primary haematological system disease or other malignancies or receive transfusion therapy during the study course.

\section{Exposure}

To evaluate the association between circulating MP TF level and VTE, patients with pancreatic cancer will be divided into two groups based on the presence or absence of VTE. The MP TF level will be compared between two groups. There are no strict restrictions on the diagnosis approach for VTE, and diagnosis by clinical assessment, imaging and D-dimer testing are all acceptable. To explore the impact of TF expression on patients' survival, patients will be defined as groups of high TF expression or low TF expression. Survival between different TF-expression groups will be compared. The TF will be detected using immunohistochemistry analysis of the tissue specimens.

\section{Outcomes}

According to different purpose, studies are eligible if they: (1) individually report the level of circulating MP
TF of patients with VTE and without VTE; or (2) report survival endpoints (including OS and progression free survival (PFS)) of patients in the groups of high TF expression and low TF expression. In addition, the level of circulating MP TF is determined by flowcytometry or activity tests. The studies reporting survival data should also provide sufficient information to estimate the HR.

\section{Studies}

Any prospective or retrospective published studies which evaluate the relationship between $\mathrm{TF}$ and pancreatic cancer are included. There is no limitation regarding the number of participants. Only studies published in English were included.

\section{Information sources}

We will search the Medline, Embase and Cochrane Library from inception to June 2020.

\section{Search strategy}

Two reviewers search the Medline, EMBASE and Cochrane using a combination of subject terms with free-text terms. The following search words are adopted for each database: (pancreas or pancrea* or pancreas $[\mathrm{MeSh}]$ ) and (carcinoma [MeSh] or adenocarcinoma [MeSh] or carcinoma, ductal [MeSh] or neoplasms [MeSh] or cancer* or carcin* or neoplas* or tumo* or cyst* or growth* or adenocarcin* or malig*) and (tissue factor or TF expression or microparticle or TF-positive MP or TF+MPor TF-bearing MP or MP-associated or MP TF or microvesicle or TF-positive MV or TF+MV or TF-bearing MV or MV-associated or MV TF or CD142 antigens or coagulation factor III or thromboplastin [MeSh] ). An example of the Medline search strategy is provided in online supplementary file 1 . Reference lists of eligible studies and relevant review articles will also be hand-searched to identify any potentially eligible studies that are recorded in the databases. We will also search Google Scholar to identify any grey literature.

\section{Study records}

\section{Data management}

All study records will be processed through EndNote X9, which can identify and remove duplicates. All extracted data will be stored in a Microsoft Excel spreadsheet.

\section{Study selection and data collection process}

Two reviewers will independently screen first the titles and abstracts of all retrieved articles after removing duplicates, and then the full texts of the selected articles. Disagreements will be resolved by face-to-face discussion, or in case of persistent disagreement, a third researcher will be consulted. The same reviewers will extract the data of included studies based on a predefined data extraction form.

\section{Data items}

The data extracted will include author, publication year, country, study design, number of participants, follow-up 
period, baseline and clinicopathological information of patients, methods used to measure TF, circulating MP TF level related to VTE presence, OS and PFS (HR, 95\% CI) related to TF expression. In addition, methods for statistical analysis, summary statistics and items associated with a risk of bias will also be summarised.

\section{Risk of bias in individual studies}

Two researchers will independently assess the quality of the each included study using the Newcastle-Ottawa Scale (NOS score). ${ }^{25}$ Scores of the NOS are split into three aspects: object selection, inter-group comparability and outcome measurement. It is generally considered that an article with a score $0-5$ point(s) is of low quality, and an article with a score $>6$ is of high quality.

\section{Data synthesis}

All statistical tests are calculated with RevMan V.5.3. The pooled SMD with $95 \%$ CIs will be used to compare the circulating MP TF level in VTE group and non-VTE group because the MP TF level reported in studies may have inconsistent units. The HR and 95\% CI of each study will be used to evaluate the survival difference between groups with different strength of TF expression. The case number and mean and SD of MP TF level are collected from included studies. The HR and 95\% CI will first be extracted from the original published study. The method from Tierney $e t a l^{26}$ is used to extract the data or conduct a variable transformation if the studies do not directly provide HR and 95\% CI. For example, some studies provide only the survival curve. The HR and $95 \% \mathrm{CI}$ of each study are finally combined using the method of generic inverse variance in RevMan software; therefore, the $\ln (\mathrm{HR})$ and SEln (HR) will be calculated in advance based on the data extracted. The level of statistical significance is set at $5 \%$. If a meta-analysis is not appropriate because of small number of studies or concerns regarding substantial variability, the result will only be reported qualitatively. The heterogeneity will be assessed using the $\mathrm{I}^{2}$ statistic. We will use the fixed-effect model when the effects are assumed to be homogenous ( $p>0.05, \mathrm{I}^{2} \leq 50 \%$ ) and the random effect model when they are heterogeneous $\left(\mathrm{p}<0.05, \mathrm{I}^{2} \geq 50 \%\right)$. To explore the source of heterogeneity, subgroup analysis will be performed according to the same regions, study design, types of tumour and methods of measuring MP TF level. Sensitivity analysis will also be conducted and focused on studies with a low risk of bias.

\section{Meta-biases}

Reporting bias will be explored graphically by a funnel plot and statistically by the Egger's test ${ }^{27}$ if 10 or more studies are available. $\mathrm{P}<0.05$ is considered to indicate publication bias.

\section{Confidence in cumulative evidence}

The Grades of Recommendation, Assessment, Development and Evaluation method is applied to assess the level of evidence obtained from this systematic review. ${ }^{28}$
Patient and public involvement

No patients are involved.

\section{DISCUSSION}

Studies have shown that TF is closely related to the VTE development and survival of patients with pancreatic cancer. ${ }^{922} 2429$ Nevertheless, no systematic review regarding the predictive value of plasma MP TF level and tissue TF expression strength on patients with pancreatic cancer have been published in English. Thus, we plan to conduct a systematic review and meta-analysis on this topic. We hope that the results of this review will provide researchers and clinicians with more convincing evidence on whether TF is a potential biomarker of thrombotic risk and prognostic factor, and thereby promote the development of individualised diagnosis and treatment of patients with pancreatic cancer. This article provides a detailed and complete description of the methodology of the review; however, some limitations may become apparent during the course of this review. First, only studies published in English will be included. The exclusion of non-English articles will negatively influence the representativeness of the results to a global population. Second, the inclusion criteria regarding the methods used to measure TF are not strict. Heterogeneity in the measuring methods will reduce the accuracy of results. Third, as with all systematic reviews and meta-analyses, there are potential risks of publication bias. Overall, regardless of the potential methodological deficiencies, we believe that each aspect of the review has been addressed and the protocol will help ensure study's integrity and transparency.

\section{ETHICS AND DISSEMINATION}

This proposed systematic review and meta-analysis is based on published data, and no information of an individual patient will be accessed and discussed in this review. Therefore, ethical approval is not required. The results of this review will be sought for publication in a peer-reviewed journal and relevant conference proceedings. Data generated during the research will be available from the corresponding author on reasonable request.

Contributors $\mathrm{HL}$ and $\mathrm{HC}$ conceived the idea and planned the entire method of undertaking this study. HL and YY wrote the protocol. YY, QS, XC and PZ designed the search strategy and planned the data extraction. DW, PT, BG, XL, TZ, LX, DX, LG and $\mathrm{PME}$ made contributions in conceiving this research project. All authors revised and approved the final version of the manuscript.

Funding This work was supported by Key Talents Project of Gansu Province (No. 2019RCXM020), Talents Innovation and Entrepreneurship Program of Lanzhou City (No. 2017-RC-62), Science and Technology Project of Chengguan District of Lanzhou City (No. 2019RCCX0034), Key Project of Science and Technology in Gansu Province(No. 19ZD2WA001), and Cuiying Scientific and Technological Innovation Program of Lanzhou University Second Hospital (No. CY2017-ZD01).

Competing interests None declared.

Patient and public involvement Patients and/or the public were not involved in the design, or conduct, or reporting, or dissemination plans of this research.

Patient consent for publication Not required. 
Provenance and peer review Not commissioned; externally peer reviewed.

Open access This is an open access article distributed in accordance with the Creative Commons Attribution Non Commercial (CC BY-NC 4.0) license, which permits others to distribute, remix, adapt, build upon this work non-commercially, and license their derivative works on different terms, provided the original work is properly cited, appropriate credit is given, any changes made indicated, and the use is non-commercial. See: http://creativecommons.org/licenses/by-nc/4.0/.

\section{ORCID iDs}

Yang Yu http://orcid.org/0000-0002-2409-3681

Hao Chen http://orcid.org/0000-0001-9741-9403

\section{REFERENCES}

1 Ferlay J, Soerjomataram I, Dikshit R, et al. Cancer incidence and mortality worldwide: sources, methods and major patterns in GLOBOCAN 2012. Int J Cancer 2015;136:E359-86.

2 Rahib L, Smith BD, Aizenberg R, et al. Projecting cancer incidence and deaths to 2030: the unexpected burden of thyroid, liver, and pancreas cancers in the United States. Cancer Res 2014:74:2913-21.

3 Chu LC, Goggins MG, Fishman EK. Diagnosis and detection of pancreatic cancer. Cancer J 2017;23:333-42.

4 Sproul EE. Carcinoma and venous thrombosis: the frequency of association of carcinoma in the body or tail of the pancreas with multiple venous thrombosis. Am J Cancer 1938;34.

5 Khorana AA, Fine RL. Pancreatic cancer and thromboembolic disease. Lancet Oncol 2004;5:655-63.

6 Campello E, llich A, Simioni $\mathrm{P}$, et al. The relationship between pancreatic cancer and hypercoagulability: a comprehensive review on epidemiological and biological issues. $\mathrm{Br} \mathrm{J}$ Cancer 2019;121:359-71.

7 Nemerson Y. Tissue factor and hemostasis. Blood 1988;71:1-8.

8 Haas SL, Jesnowski R, Steiner M, et al. Expression of tissue factor in pancreatic adenocarcinoma is associated with activation of coagulation. World J Gastroenterol 2006;12:4843-9.

9 Khorana AA, Ahrendt SA, Ryan CK, et al. Tissue factor expression, angiogenesis, and thrombosis in pancreatic cancer. Clin Cancer Res 2007;13:2870-5.

10 Khorana AA, Francis CW, Menzies KE, et al. Plasma tissue factor may be predictive of venous thromboembolism in pancreatic cancer. $J$ Thromb Haemost 2008:6:1983-5.

11 Wang J-G, Geddings JE, Aleman MM, et al. Tumor-Derived tissue factor activates coagulation and enhances thrombosis in a mouse xenograft model of human pancreatic cancer. Blood 2012;119:5543-52

12 Tesselaar MET, Romijn FPHTM, Van Der Linden IK, et al. Microparticle-Associated tissue factor activity: a link between cancer and thrombosis? J Thromb Haemost 2007;5:520-7.

13 Unruh D, Sagin F, Adam M, et al. Levels of alternatively spliced tissue factor in the plasma of patients with pancreatic cancer may help predict aggressive tumor phenotype. Ann Surg Oncol 2015;22:1206-11.

14 Davila M, Robles-Carrillo L, Unruh D, et al. Microparticle association and heterogeneity of tumor-derived tissue factor in plasma: is it important for coagulation activation? J Thromb Haemost 2014;12:186-96.

15 Bharthuar A, Khorana AA, Hutson A, et al. Circulating microparticle tissue factor, thromboembolism and survival in pancreaticobiliary cancers. Thromb Res 2013;132:180-4.

16 Boone BA, Zenati MS, Rieser C, et al. Risk of venous thromboembolism for patients with pancreatic ductal adenocarcinoma undergoing preoperative chemotherapy followed by surgical resection. Ann Surg Oncol 2019;26:1503-11.

17 Mier-Hicks A, Raj M, Do RK, et al. Incidence, management, and implications of visceral thrombosis in pancreatic ducta adenocarcinoma. Clin Colorectal Cancer 2018;17:121-8.

18 Milsom CC, Yu JL, Mackman N, et al. Tissue factor regulation by epidermal growth factor receptor and epithelial-to-mesenchymal transitions: effect on tumor initiation and angiogenesis. Cancer Res 2008;68:10068-76.

19 Date K, Ettelaie C, Maraveyas A. Tissue factor-bearing microparticles and inflammation: a potential mechanism for the development of venous thromboembolism in cancer. J Thromb Haemost 2017;15:2289-99.

20 Hu C, Huang L, Gest C, et al. Opposite regulation by PI3K/Akt and MAPK/ERK pathways of tissue factor expression, cell-associated procoagulant activity and invasiveness in MDA-MB-231 cells. $J$ Hematol Oncol 2012;5:16.

21 Chanakira A, Westmark PR, Ong IM, et al. Tissue factor-factor VIla complex triggers protease activated receptor 2-dependent growth factor release and migration in ovarian cancer. Gynecol Oncol 2017;145:167-75.

22 Chen K, Li Z, Jiang P, et al. Co-Expression of CD133, CD44v6 and human tissue factor is associated with metastasis and poor prognosis in pancreatic carcinoma. Oncol Rep 2014;32:755-63.

23 Kakkar AK, Lemoine NR, Scully MF, et al. Tissue factor expression correlates with histological grade in human pancreatic cancer. $\mathrm{Br} \mathrm{J}$ Surg 1995;82:1101-4.

24 Nitori N, Ino Y, Nakanishi Y, et al. Prognostic significance of tissue factor in pancreatic ductal adenocarcinoma. Clin Cancer Res 2005;11:2531-9.

25 Stang A. Critical evaluation of the Newcastle-Ottawa scale for the assessment of the quality of nonrandomized studies in metaanalyses. Eur J Epidemiol 2010;25:603-5.

26 Tierney JF, Stewart LA, Ghersi D, et al. Practical methods for incorporating summary time-to-event data into meta-analysis. Trials 2007;8:16.

27 Duval S, Tweedie R. Trim and fill: a simple funnel-plot-based method of testing and adjusting for publication bias in meta-analysis. Biometrics 2000;56:455-63.

28 Iorio A, Spencer FA, Falavigna M, et al. Use of grade for assessment of evidence about prognosis: rating confidence in estimates of event rates in broad categories of patients. BMJ 2015;350:h870.

29 Geddings JE, Mackman N. Tumor-Derived tissue factor-positive microparticles and venous thrombosis in cancer patients. Blood 2013;122:1873-80. 